\title{
Stress-energy tensor of quantized massive fields in static wormhole spacetimes
}

\author{
Ewa Kocuper, Jerzy Matyjasek* and Kasia Zwierzchowska \\ Institute of Physics, Maria Curie-Sktodowska University \\ pl. Marii Curie-Sktodowskiej 1, 20-031 Lublin, Poland
}

\begin{abstract}
In order to be traversable, the static Lorentzian wormhole must be made out of some exotic matter that violates the weak energy condition. The quantized fields are the natural candidates as their stress-energy tensor, in many cases, possesses desired properties. In this paper we construct and examine the stress-energy tensor of the quantized massive scalar, spinor and vector fields in six static wormhole spacetimes. We find that in all considered cases the quantum fields violate the Morris-Thorne conditions and do not have the form necessary to support the wormhole throat. This is in concord with the previous results and indicates that the massive quantum fields make the wormholes less operable.
\end{abstract}

PACS numbers: 04.62.+v,04.70.-s

\section{INTRODUCTION}

Since their invention the Lorentzian wormholes have been a subject of intensive studies [1, 2]. Treated as shortcuts in a spacetime these strange solutions of the Einstein field equations opened the discussion of interstellar or even intergalactic journeys. Moreover, careful analyses of the casual structure of the spacetimes of the static wormholes indicated the possibility of the existence of closed timelike curves that lead to the inevitable time-machine interpretation of such configurations. Surely such strange objects are hard to construct in practice: it was shown that the stress-energy tensor of the wormhole has to satisfy the more or less exotic conditions, excluding the possibility of constructing an operating wormhole out of any known form of the ordinary classical matter. On the other hand however, it is a well-known fact that the quantized fields may violate classical energy conditions and therefore may appear to be of sufficient exoticity to maintain a wormhole.

Having an operating traversable wormhole may be a source of problems. Indeed, it would not only be an interesting and perhaps useful device for us, but also it could play a similar role for some advanced civilization. Following this line of reasoning one can regard the fundamental obstacles one encounters in projecting and constructing wormholes as rather fortunate.

To be a good wormhole the solution of the Einstein field equations must satisfy some quite

\footnotetext{
* email: jurek@kft.umcs.lublin.pl
} 
restrictive requirements, such as the existence of a throat that connects two spacetime regions, the nonexistence of the event horizon, traversability by humans (that sets the scale for the curvature) and perturbative stability. There have been numerous attempts to construct the exact solutions of the Einstein field equations describing a traversable wormhole. Various features of such solutions are well documented and have been analyzed in numerous papers, e.g., in Refs. [3 6] (and the references cited therein). On the other hand however, less is known about wormholes generated or influenced by the quantized fields. This is perhaps not particularly strange as the calculations of this sort are very complicated and have to be carried out with the aid of numerical methods. On the other hand however, one may, as a preliminary step, construct and analyze the renormalized stress-energy tensor of the quantized fields of various types in a spacetime of interest, and decide if the obtained $\left\langle T_{a}^{b}\right\rangle$ has the appropriate form to support a traversable wormhole spacetime. Such calculations have been initiated by Taylor et al. in Ref [7], where the stress-energy tensor of the quantized massive scalar field has been constructed and analyzed for the five types of wormhole spacetimes (types A - E of Sect. IV]. They used the general formula constructed in Ref. [8] that is valid for an arbitrary static and spherically symmetric spacetime and concluded that for both minimally and conformally coupled massive scalar fields the stress-energy tensor does not have the form needed to support the wormhole geometry and that the exotic conditions were satisfied for clearly unphysical values of the coupling constant. Other results, especially those obtained in Refs. [9 16] were devoted to various aspects of the quantum field theory in the wormhole spacetimes. In this regard the (numerical) construction of the semiclassical wormhole reported in Ref. [17] is of principal interest.

The analytic approximation used in Ref. [8] is based on the WKB approximation of the solutions of the massive scalar field equation in the spherically symmetric spacetime and the summation of the thus obtained modes by means of the Abel-Plana method. In the large-mass limit it is equivalent to the Schwinger-DeWitt expansion based on the heat kernel expansion [18 22]; to obtain the lowest (i. e. $\mathrm{m}^{-2}$ ) terms, one has to use the sixth-order WKB approximation. The same result can be obtained within the framework of the Schwinger-DeWitt approach. Now one has to retain the coincidence limit of the first nontrivial Hadamard-DeWitt coefficient, $a_{3}\left(x, x^{\prime}\right)$. Numerical calculations reported in Ref. [23] indicated that the Schwinger-DeWitt method provides a good approximation of the renormalized stress-energy tensor of the massive scalar field with an arbitrary curvature coupling as long as the mass of the field remains sufficiently large.

Some time ago we calculated the approximate renormalized $\left\langle T_{a}^{b}\right\rangle$ of the massive scalar, spinor and vector fields in an arbitrary spacetime functionally differentiating the effective action (con- 
structed from the coincidence limit of Hadamard-DeWitt coefficient $a_{3}$ ) with respect to the metric tensor [21, 22]. (The next-to-leading term calculated from the coefficient $a_{4}$ has been presented in Ref. 24] and the generalization of the method to the higher dimensions has been discussed in Refs. [25 27].) The resulting stress-energy tensor is rather involved and consists of a few dozen terms, such as terms cubic in curvature or involving its fourth covariant derivatives. The obtained $\left\langle T_{a}^{b}\right\rangle$ generalizes the results derived in Ref. [20] by Frolov and Zel'nikov for vacuum type-D geometries. It is expected that for sufficiently massive fields, i.e., where the Compton length is much smaller than the characteristic radius of curvature, the approximation is quite reasonable. The usual argument in this regard is that in such a case the nonlocal contribution to the total effective action may be neglected.

In this paper we shall extend the analyses of Ref. [7] to the case of the massive spinor and vector fields and construct the renormalized stress-energy tensor in the six models of the traversable wormholes. The stress-energy tensor of the quantized massive scalar field in the first five configurations has been constructed earlier. We believe our spinor and vector results as well as the result obtained for the wormhole supported by the phantom energy are new. Although the stress-energy tensor of the quantized fields constructed in the wormhole spacetime is of great interest and importance in its own right, we will extend our discussion to the question of whether the quantized matter is of sufficient exoticity to support the traversable wormhole. Ideally, the problem should be treated within the framework of the semiclassical gravity and it seems that the stress-energy tensor constructed within the framework of the Schwinger-DeWitt approximation is very well suited for this. Indeed, it depends functionally on a general metric and can be used in virtually any spacetime provided the above-mentioned conditions are satisfied. This, however, would require the numerical analysis of the complex system of the sixth-order differential equations in the spirit of Ref. [17] that is beyond the scope of the present paper. Instead, we will confine ourselves to the analysis of the properties of the stress-energy tensor at the throat and its closest vicinity. The general expressions describing the stress-energy tensor are usually too long, complicated and not particularly illuminating and will be not presented here. ${ }^{1}$

The paper is organized as follows. In Sec. II the general Morris-Thorne conditions are introduced. In Sec. III we discuss the general stress-energy tensor of the quantized massive fields in the large-mass limit. The analysis of the stress-energy tensor and the Morris-Thorne conditions in the

\footnotetext{
${ }^{1}$ The general expressions describing the stress-energy tensor of the quantized massive scalar, spinor and vector fields can be obtained on request from the second author.
} 
six exemplary wormhole spacetimes (cases A - F) are presented in Sec. IV. Finally, Sec. V contains summary and a brief discussion of possible extensions and generalizations of the presented results.

\section{THE GENERAL MORRIS-THORNE WORMHOLE}

The line element of the static and spherically symmetric wormhole is given by

$$
d s^{2}=-\exp (2 \phi) d t^{2}+\left(1-\frac{b}{r}\right)^{-1} d r^{2}+r^{2}\left(d \theta^{2}+\sin ^{2} \theta d \varphi^{2}\right),
$$

where $\phi(r)$ and $b(r)$ are the redshift and the shape functions, respectively. The radial coordinate decreases from infinity to its minimal value $r=r_{0}$ (describing the throat) and subsequently it goes to infinity.

Simple calculations show that in the static orthonormal frame the Einstein field equations can be written in the form

$$
\rho=T_{\hat{t} \hat{t}}=\frac{1}{8 \pi r^{2}} \frac{d}{d r} b
$$

and

$$
\tau=-T_{\hat{r} \hat{r}}=\frac{1}{8 \pi}\left[\frac{b}{r^{3}}-\frac{2}{r}\left(1-\frac{b}{r}\right) \frac{d}{d r} \phi\right]
$$

The equations for the mass-energy, $\rho$, and the radial tension, $\tau$, are supplemented by the third equation

$$
p=\frac{r}{2}\left[(\rho-\tau) \frac{d}{d r} \phi-\frac{d}{d r} \tau\right]
$$

for the lateral pressure $p=T_{\hat{\varphi} \hat{\varphi}}=T_{\hat{\theta} \hat{\theta}}$. The traversable wormhole configurations require satisfying at the throat (located at $r=r_{0}$ ) the following minimal set of conditions

$$
\tau_{0}>0
$$

and

$$
\frac{\tau_{0}-\rho_{0}}{\left|\rho_{0}\right|} \geq 0
$$

The first condition follows (via the Einstein field equations) from the finiteness of $\rho_{0}$ and from the absence of the event horizon. The second inequality is the flaring-out condition [1].

Now, let us assume that we have the solution of the Einstein field equations describing an operating wormhole and consider quantum fields in the spirit of the quantum field theory in curved 
background, i.e., the mean value of the stress-energy tensor of the quantum fields does not contribute to the source term. In general, depending on its characteristic, the action of the quantum field can be either destructive or constructive. Indeed, if the Morris-Thorne conditions are not satisfied, the quantum effects tend to destroy or at least make the wormhole less functional. In the next section we shall construct the stress-energy tensors of the massive scalar, spinor and vector fields in the spacetimes of the six wormhole configurations.

\section{STRESS-ENERGY TENSOR OF THE MASSIVE SCALAR, SPINOR AND VECTOR FIELDS}

The approximate one-loop effective action of the quantized massive scalar, spinor and vector fields is given by

$$
\begin{aligned}
W^{(1)} & =\frac{1}{192 \pi^{2} m_{\star}^{2}} \int d^{4} x g^{1 / 2}\left[\alpha_{1} R \square R+\alpha_{2} R_{p q} \square R^{p q}+\alpha_{3} R^{3}\right. \\
& +\alpha_{4} R R_{p q} R^{p q}+\alpha_{5} R R_{p q a b} R^{p q a b}+\alpha_{6} R_{q}^{p} R_{a}^{q} R_{p}^{a}+\alpha_{7} R^{p q} R_{a b} R_{p q}^{a b} \\
& \left.+\alpha_{8} R_{p q} R_{c a b}^{p} R^{q c a b}+\alpha_{9} R_{a b}{ }^{p q} R_{p q}{ }^{c d} R_{c d}^{a b}+\alpha_{10} R_{p q}^{a b} R_{c d^{p}{ }^{q} R_{a b}^{c} d}\right] \\
& =\frac{1}{192 \pi^{2} m_{\star}^{2}} \sum_{i=1}^{10} \alpha_{i} W_{i},
\end{aligned}
$$

where $m_{\star}$ is the effective mass and

$$
\alpha_{i}=\frac{\alpha_{i}^{(0)}}{n_{0}}+\frac{\alpha_{i}^{(1 / 2)}}{n_{1 / 2}}+\frac{\alpha_{i}^{(1)}}{n_{1}} .
$$

The coefficients $\alpha_{i}^{(s)}$ are tabulated in Table I and the parameters $n_{s}$ are defined by the simple relations $m_{0}^{2}=n_{0} m_{\star}^{2}, m_{1 / 2}^{2}=n_{1 / 2} m_{\star}^{2}$ and $m_{1}^{2}=n_{1} m_{\star}^{2}$. The $s$-spin field can be excluded from the calculations by taking the limit $n_{s} \rightarrow \infty$. Without loss of generality the parameter $n_{0}$ can be normalized by taking $m_{\star}$ to be the effective mass of the scalar fields, i.e., $n_{0}=1$ if the massive scalar field is present and $n_{0}=\infty$ if it is absent.

The renormalized stress-energy tensor is given by

$$
\left\langle T^{a b}\right\rangle=\frac{2}{g^{1 / 2}} \frac{\delta}{\delta g_{a b}} W_{r e n}=\frac{1}{96 \pi^{2} m_{\star}^{2} g^{1 / 2}} \sum_{i=1}^{10} \alpha_{i} \frac{\delta}{\delta g_{a b}} W_{i}
$$

and the full form of the functional derivatives of $W_{i}$ with respect to the metric tensor was given in Refs. [21, 22]. It is convenient to rewrite the stress-energy tensor in the following form

$$
\left\langle T_{a}^{b}\right\rangle=\frac{1}{96 \pi^{2} m_{\star}^{2}}\left(\frac{t_{a}^{b}}{n_{0}}+\frac{s_{a}^{b}}{n_{1 / 2}}+\frac{v_{a}^{b}}{n_{1}}\right),
$$


TABLE I: The coefficients $\alpha_{i}^{(s)}$ for the massive scalar, spinor, and vector fields

\begin{tabular}{|c|c|c|c|}
\hline \hline & $\mathrm{s}=0$ & $\mathrm{~s}=1 / 2$ & $\mathrm{~s}=1$ \\
\hline$\alpha_{1}^{(s)}$ & $\frac{1}{2} \xi^{2}-\frac{1}{5} \xi+\frac{1}{56}$ & $-\frac{3}{140}$ & $-\frac{27}{280}$ \\
$\alpha_{2}^{(s)}$ & $\frac{1}{140}$ & $\frac{1}{14}$ & $\frac{9}{28}$ \\
$\alpha_{3}^{(s)}$ & $\left(\frac{1}{6}-\xi\right)^{3}$ & $\frac{1}{432}$ & $-\frac{5}{72}$ \\
$\alpha_{4}^{(s)}$ & $-\frac{1}{30}\left(\frac{1}{6}-\xi\right)$ & $-\frac{1}{90}$ & $\frac{31}{60}$ \\
$\alpha_{5}^{(s)}$ & $\frac{1}{30}\left(\frac{1}{6}-\xi\right)$ & $-\frac{7}{720}$ & $-\frac{1}{10}$ \\
$\alpha_{6}^{(s)}$ & $-\frac{8}{945}$ & $-\frac{25}{378}$ & $-\frac{52}{63}$ \\
$\alpha_{7}^{(s)}$ & $\frac{2}{315}$ & $\frac{47}{630}$ & $-\frac{19}{105}$ \\
$\alpha_{8}^{(s)}$ & $\frac{1}{1260}$ & $\frac{19}{630}$ & $\frac{61}{140}$ \\
$\alpha_{9}^{(s)}$ & $\frac{17}{7560}$ & $\frac{29}{3780}$ & $-\frac{67}{2520}$ \\
$\alpha_{10}^{(s)}$ & $-\frac{1}{270}$ & $-\frac{1}{54}$ & $\frac{1}{18}$ \\
\hline \hline
\end{tabular}

where $t_{a}^{b}, s_{a}^{b}$ and $v_{a}^{b}$ are the purely geometric parts of the stress-energy tensor of the scalar, spinor and vector fields, respectively.

Using ordinary tensor components rather than proper reference frame components one has

$$
\begin{gathered}
\rho=-\left\langle T_{t}^{t}\right\rangle, \\
\tau=-\left\langle T_{r}^{r}\right\rangle
\end{gathered}
$$

and

$$
p=\left\langle T_{\theta}^{\theta}\right\rangle=\left\langle T_{\phi}^{\phi}\right\rangle
$$

Our analysis will be confined to the closest vicinity of the throat. We will concentrate on the scalar, vector and spinor quantum fields propagating in the five wormhole spacetimes (cases A E) considered earlier by Taylor et al. Additionally, we will also consider the case of the wormhole supported by phantom energy [28]. It should be noted that for the scalar fields with arbitrary curvature our approach gives precisely the same results as these presented in Ref. [7] and for the reader's convenience we shall also display them in this paper. We only remark that taking into account fundamental differences of the two methods the equality of the results, although expected, is indeed really impressive.

We conclude this section with a few words on implementation. The functional derivatives of the effective action (7) with respect to the general metric tensor as well as basic simplifications of the thus obtained result have been constructed with the aid of the FORM [29, 30]. The result has 
been converted into the Mathematica and Maple syntaxes for further tensor simplifications and for calculations in the local maps. The final result has also been derived

from the effective action constructed from the full form of the coefficient $\left[a_{3}\right]$. In both cases the FORM calculations have been successfully executed within a fraction of a second.

\section{QUANTUM FIELDS AND THE MORRIS-THORNE CONDITIONS}

Now we shall construct the stress-energy tensor of the quantized fields in a large-mass limit in six wormhole spacetimes and check if it satisfies the conditions (5) and (6). In general, the stress-energy tensor for the spacetime described by the line element (1) is quite complicated and to avoid the presentation of the unnecessary complex formulas we will focus on its value at the throat. We shall start our survey with the simplest case of the zero-tidal wormhole.

\section{A. Zero-tidal wormhole}

The simplest traversable wormhole with the zero radial tides is described by the line element (1) with

$$
\phi(r)=0, \quad b(r)=r_{0}
$$

Because of its form one expects massive simplifications in the stress-energy tensor. Indeed, for the scalar, spinor and vector fields, after some algebra, one has

$$
\begin{aligned}
\left\langle T_{t}^{t}\right\rangle & =\frac{1}{96 \pi^{2} m_{\star}^{2} r_{0}^{6}}\left(\frac{43-252 \xi}{560 n_{0}}-\frac{3}{56 n_{1 / 2}}-\frac{53}{560 n_{1}}\right), \\
\left\langle T_{r}^{r}\right\rangle & =\frac{1}{96 \pi^{2} m_{\star}^{2} r_{0}^{6}}\left(\frac{23-84 \xi}{560 n_{0}}+\frac{3}{70 n_{1 / 2}}+\frac{83}{560 n_{1}}\right)
\end{aligned}
$$

and

$$
\left\langle T_{\theta}^{\theta}\right\rangle=\frac{1}{96 \pi^{2} m_{\star}^{2} r_{0}^{6}}\left(+\frac{5-21 \xi}{56 n_{0}}+\frac{33}{560 n_{1 / 2}}+\frac{19}{280 n_{1}}\right) .
$$

Although the maximal power of $\xi$ is 3 (as can be seen from Table I) the stress-energy tensor is linear in $\xi$. It is because the curvature scalar vanishes, and, consequently, the first and third terms of (7) do not contribute to the final result.

The Morris-Thorne conditions require the tension at the throat to be positive, i.e., $\tau_{0}>0$. Moreover, in order to construct the traversable wormhole the tension should be greater than or 
equal to the energy density, $\tau_{0} \geq \rho_{0}$. Inspection of the stress-energy tensor shows that the tension of the spinor and vector fields is negative whereas the energy density is positive. It follows then that both conditions are violated and the spinor and vector fields tend to destroy the wormhole. The analysis carried out in Ref. [7] showed that the Morris-Thorne conditions cannot be satisfied simultaneously by the scalar field. Indeed, from Eqs. (15) and (16) one concludes that the tension is positive for $\xi>23 / 84$ and the condition (6) is satisfied for $\xi \leq 5 / 42$.

\section{B. The simple wormhole}

As a second example let us consider a slightly more complicated ultrastatic wormhole with

$$
\phi(r)=0, \quad b(r)=\frac{r_{0}^{2}}{r} .
$$

Being still relatively simple, the stress-energy tensor at the throat can be written in the following compact form

$$
\begin{gathered}
\left\langle T_{t}^{t}\right\rangle=-\frac{1}{96 \pi^{2} m_{\star}^{2} r_{0}^{6}}\left(\frac{19320 \xi^{3}-33180 \xi^{2}+11242 \xi-1033}{210 n_{0}}+\frac{19}{21 n_{1 / 2}}+\frac{381}{70 n_{1}}\right), \\
\left\langle T_{r}^{r}\right\rangle=-\frac{1}{96 \pi^{2} m_{\star}^{2} r_{0}^{6}}\left(\frac{4200 \xi^{3}-5460 \xi^{2}+1806 \xi-187}{210 n_{0}}-\frac{23}{105 n_{1 / 2}}-\frac{89}{70 n_{1}}\right)
\end{gathered}
$$

and

$$
\left\langle T_{\theta}^{\theta}\right\rangle=-\frac{1}{96 \pi^{2} m_{\star}^{2} r_{0}^{6}}\left(\frac{19320 \xi^{3}-33180 \xi^{2}+11634 \xi-1213}{210 n_{0}}-\frac{131}{105 n_{1 / 2}}-\frac{527}{70 n_{1}}\right) .
$$

The energy density of the massive scalar field is positive for $0.1622 \leq \xi \leq 0.2532$ and for $\xi \geq 1.302$ whereas the radial tension is negative for $\xi<0.8604$. Finally, the lateral pressure is positive for $\xi \leq 1.2876$. Now, let us return to the Morris-Thorne conditions and consider the spinor and vector fields first. The condition (5) is violated as the radial tension in both cases is negative. Similarly, since $\rho_{0}$ is positive, the second condition is also violated. The case of the scalar fields is slightly more complicated: simple manipulations indicate that the Morris-Thorne conditions are satisfied for (approximately) $0.8604 \leq \xi \leq 1.4222$.

\section{The "absurdly benign" wormhole}

The wormhole configurations considered in this subsection are characterized by the locality of the distribution of the exotic matter. The matter is confined to an arbitrary small region in the 
closest vicinity of the throat, i.e.,

$$
\phi(r)=0, \quad b(r)=\frac{r_{0}\left(a+r_{0}-r\right)^{2}}{a^{2}}
$$

for $r_{0} \leq r<r_{0}+a$ and $b(r)=0$ outside this region.

Adopting the notation of Sec. III (Eq. 10) the (rescaled) time component of the stress-energy tensor in each of the considered cases is given by

$$
\begin{aligned}
& t_{t}^{t}=\frac{\xi^{3}\left(-161280 a^{3} r_{0}^{2}-349440 a^{2} r_{0}^{3}-161280 a r_{0}^{4}\right)}{1680 a^{5} r_{0}^{6}} \\
&+\frac{\xi^{2}\left(23520 a^{4} r_{0}+191520 a^{3} r_{0}^{2}+342720 a^{2} r_{0}^{3}+164640 a r_{0}^{4}+6720 r_{0}^{5}\right)}{1680 a^{5} r_{0}^{6}} \\
&+\frac{\xi\left(-756 a^{5}-11536 a^{4} r_{0}-59080 a^{3} r_{0}^{2}-95200 a^{2} r_{0}^{3}-46368 a r_{0}^{4}-2688 r_{0}^{5}\right)}{1680 a^{5} r_{0}^{6}} \\
&+ \frac{129 a^{5}+1202 a^{4} r_{0}+5134 a^{3} r_{0}^{2}+7856 a^{2} r_{0}^{3}+3884 a r_{0}^{4}+264 r_{0}^{5}}{1680 a^{5} r_{0}^{6}}, \\
& s_{t}^{t}=-\frac{45 a^{5}+334 a^{4} r_{0}+668 a^{3} r_{0}^{2}+632 a^{2} r_{0}^{3}+230 a r_{0}^{4}+12 r_{0}^{5}}{840 a^{5} r_{0}^{6}}
\end{aligned}
$$

and

$$
v_{t}^{t}=-\frac{159 a^{5}+2302 a^{4} r_{0}+7026 a^{3} r_{0}^{2}+9584 a^{2} r_{0}^{3}+3972 a r_{0}^{4}+216 r_{0}^{5}}{1680 a^{5} r_{0}^{6}}
$$

Similarly, for the radial and angular components one has

$$
\begin{aligned}
& t_{r}^{r}=\frac{\xi^{3}\left(-80640 a^{2} r_{0}^{2}-107520 a r_{0}^{3}\right)}{1680 a^{4} r_{0}^{6}} \\
&+ \frac{\xi^{2}\left(6720 a^{3} r_{0}+70560 a^{2} r_{0}^{2}+94080 a r_{0}^{3}+13440 r_{0}^{4}\right)}{1680 a^{4} r_{0}^{6}} \\
&+ \frac{\xi\left(-252 a^{4}-3360 a^{3} r_{0}-19824 a^{2} r_{0}^{2}-25536 a r_{0}^{3}-5376 r_{0}^{4}\right)}{1680 a^{4} r_{0}^{6}} \\
&+ \frac{69 a^{4}+450 a^{3} r_{0}+1878 a^{2} r_{0}^{2}+2272 a r_{0}^{3}+552 r_{0}^{4}}{1680 a^{4} r_{0}^{6}} \\
& s_{r}^{r}=\frac{36 a^{4}+96 a^{3} r_{0}+201 a^{2} r_{0}^{2}+164 a r_{0}^{3}+36 r_{0}^{4}}{840 a^{4} r_{0}^{6}} \\
& v_{r}^{r}=\frac{83 a^{4}+254 a^{3} r_{0}+842 a^{2} r_{0}^{2}+928 a r_{0}^{3}+216 r_{0}^{4}}{560 a^{4} r_{0}^{6}}
\end{aligned}
$$


and

$$
\begin{gathered}
t_{\theta}^{\theta}=\frac{\xi^{3}\left(-60480 a^{3} r_{0}^{2}-215040 a^{2} r_{0}^{3}-80640 a r_{0}^{4}\right)}{840 a^{5} r_{0}^{6}} \\
+\frac{\xi^{2}\left(10080 a^{4} r_{0}+84840 a^{3} r_{0}^{2}+198240 a^{2} r_{0}^{3}+85680 a r_{0}^{4}+3360 r_{0}^{5}\right)}{840 a^{5} r_{0}^{6}} \\
+\frac{\xi\left(-315 a^{5}-5838 a^{4} r_{0}-29484 a^{3} r_{0}^{2}-55776 a^{2} r_{0}^{3}-25200 a r_{0}^{4}-1344 r_{0}^{5}\right)}{840 a^{5} r_{0}^{6}} \\
+\frac{75 a^{5}+876 a^{4} r_{0}+3198 a^{3} r_{0}^{2}+5132 a^{2} r_{0}^{3}+2325 a r_{0}^{4}+138 r_{0}^{5}}{840 a^{5} r_{0}^{6}} \\
s_{\theta}^{\theta}=\frac{99 a^{5}+906 a^{4} r_{0}+1803 a^{3} r_{0}^{2}+1732 a^{2} r_{0}^{3}+654 a r_{0}^{4}+36 r_{0}^{5}}{1680 a^{5} r_{0}^{6}} \\
v_{\theta}^{\theta}=\frac{19 a^{5}+512 a^{4} r_{0}+1546 a^{3} r_{0}^{2}+2164 a^{2} r_{0}^{3}+939 a r_{0}^{4}+54 r_{0}^{5}}{280 a^{5} r_{0}^{6}}
\end{gathered}
$$

In this case the stress-energy tensor of the quantized massive spinor and vector fields does not satisfy the Morris-Thorne conditions as the energy density (and the lateral pressure) treated as a function of $a / r_{0}$ is always positive whereas the radial tension is always negative. On the other hand, for the massive scalar fields there is a region in the parameter space where the conditions are satisfied. However, it is possible only for very exotic and apparently unphysical values of the curvature coupling constant.

\section{Wormhole with finite radial cutoff}

This is the class of the wormhole geometries in which the zero-tidal-force solution is joined with the Schwarzschild solution at some finite radius, $a$. The line element in the vicinity of the throat $\left(r_{0} \leq r \leq a\right)$ is

$$
\phi(r)=0, \quad b(r)=r_{0}\left(\frac{r}{r_{0}}\right)^{(1-\eta)},
$$

where $0<\eta<1$. The stress-energy tensor at the throat is given by

$$
\begin{aligned}
t_{t}^{t} & =\frac{132 \eta^{5}+403 \eta^{4}-92 \eta^{3}-490 \eta^{2}+112 \eta+64}{1680 r_{0}^{6}} \\
& -\frac{4(\eta-1)^{2}\left(3 \eta^{2}+5 \eta+1\right) \xi^{3}}{r_{0}^{6}} \\
& +\frac{(\eta-1)\left(2 \eta^{4}+13 \eta^{3}+10 \eta^{2}-18 \eta-2\right) \xi^{2}}{r_{0}^{6}} \\
& -\frac{\left(48 \eta^{5}+177 \eta^{4}-41 \eta^{3}-245 \eta^{2}+64 \eta+24\right) \xi}{60 r_{0}^{6}},
\end{aligned}
$$




$$
s_{t}^{t}=-\frac{6 \eta^{5}+25 \eta^{4}+20 \eta^{3}+14 \eta-20}{840 r_{0}^{6}}
$$

and

$$
v_{t}^{t}=-\frac{36 \eta^{5}+143 \eta^{4}+36 \eta^{3}-210 \eta^{2}+112 \eta-64}{560 r_{0}^{6}} .
$$

For the radial component of the stress-energy tensor one has

$$
\begin{aligned}
t_{r}^{r} & =\frac{69 \eta^{4}+146 \eta^{3}-210 \eta^{2}+64}{1680 r_{0}^{6}} \\
& -\frac{4(\eta-1)^{2}(2 \eta+1) \xi^{3}}{r_{0}^{6}} \\
& +\frac{\left(\eta^{4}+5 \eta^{3}-8 \eta^{2}+2\right) \xi^{2}}{r_{0}^{6}} \\
& -\frac{\left(8 \eta^{4}+22 \eta^{3}-35 \eta^{2}+8\right) \xi}{20 r_{0}^{6}} \\
& s_{r}^{r}=\frac{9 \eta^{4}+23 \eta^{3}+40}{1680 r_{0}^{6}} \\
v_{r}^{r}= & \frac{27 \eta^{4}+62 \eta^{3}-70 \eta^{2}+64}{560 r_{0}^{6}}
\end{aligned}
$$

Finally, the angular component is given by

$$
\begin{aligned}
t_{\theta}^{\theta} & =\frac{138 \eta^{5}+495 \eta^{4}-19 \eta^{3}-840 \eta^{2}+504 \eta-128}{1680 r_{0}^{6}} \\
& -\frac{2(\eta-1)^{2}\left(6 \eta^{2}+13 \eta-4\right) \xi^{3}}{r_{0}^{6}} \\
& +\frac{\left(4 \eta^{5}+23 \eta^{4}-\eta^{3}-64 \eta^{2}+46 \eta-8\right) \xi^{2}}{2 r_{0}^{6}} \\
- & \frac{\left(32 \eta^{5}+130 \eta^{4}-11 \eta^{3}-280 \eta^{2}+176 \eta-32\right) \xi}{40 r_{0}^{6}} \\
& s_{\theta}^{\theta}=\frac{36 \eta^{5}+141 \eta^{4}+97 \eta^{3}+84 \eta-160}{3360 r_{0}^{6}}
\end{aligned}
$$

and

$$
v_{\theta}^{\theta}=\frac{54 \eta^{5}+201 \eta^{4}+23 \eta^{3}-280 \eta^{2}+168 \eta-128}{560 r_{0}^{6}} .
$$

Inspection of the formulas $(32,41)$ shows that the spinor and vector tend to destroy the wormhole. The radial tension is always negative as there are no roots in the interval $0 \leq \eta \leq 1$. Similarly, although in the case of the scalar field the parameter space is two-dimensional there are no configurations that support wormholes. 


\section{E. The proximal Schwarzschild wormhole}

Now, let us consider spacetime that is similar to the Schwarzschild solution with one notable modification: the $g_{t t}$ component of the metric tensor is given by

$$
g_{t t}=-\left(1-\frac{r_{0}}{r}+\frac{\varepsilon}{r^{2}}\right) \text {, }
$$

$b(r)=r_{0}$ and the line element takes the form

$$
d s^{2}=-\left(1-\frac{r_{0}}{r}+\frac{\varepsilon}{r^{2}}\right) d t^{2}+\left(1-\frac{r_{0}}{r}\right)^{-1} d r^{2}+r^{2}\left(d \theta^{2}+\sin ^{2} \theta d \varphi^{2}\right) .
$$

The role of the small positive parameter $\varepsilon$ is to prevent the existence of the event horizon and to

make the wormhole traversable. Now, making use of the general formulas of Refs. 21, 22, one gets

$$
\begin{aligned}
t_{t}^{t} & =-\frac{\xi^{3}\left(13 r_{0}^{6}-54 r_{0}^{4} \epsilon+24 r_{0}^{2} \epsilon^{2}+64 \epsilon^{3}\right)}{8 r_{0}^{6} \epsilon^{3}} \\
& +\frac{\xi^{2}\left(47 r_{0}^{6}-150 r_{0}^{4} \epsilon-22 r_{0}^{2} \epsilon^{2}-68 \epsilon^{3}\right)}{16 r_{0}^{6} \epsilon^{3}} \\
& +\frac{\xi\left(-243 r_{0}^{6}+738 r_{0}^{4} \epsilon+238 r_{0}^{2} \epsilon^{2}+400 \epsilon^{3}\right)}{240 r_{0}^{6} \epsilon^{3}} \\
& -\frac{-358 r_{0}^{6}+1044 r_{0}^{4} \epsilon+491 r_{0}^{2} \epsilon^{2}+442 \epsilon^{3}}{3360 r_{0}^{6} \epsilon^{3}} \\
s_{t}^{t} & =-\frac{-269 r_{0}^{6}+486 r_{0}^{4} \epsilon+822 r_{0}^{2} \epsilon^{2}+1396 \epsilon^{3}}{6720 r_{0}^{6} \epsilon^{3}}
\end{aligned}
$$

and

$$
v_{t}^{t}=-\frac{-1074 r_{0}^{6}+1620 r_{0}^{4} \epsilon+3223 r_{0}^{2} \epsilon^{2}+7738 \epsilon^{3}}{3360 r_{0}^{6} \epsilon^{3}} .
$$

Similarly, for the radial and angular components of the stress-energy tensor one has

$$
\begin{aligned}
t_{r}^{r} & =-\frac{\xi^{3}\left(r_{0}^{2}-2 \epsilon\right)^{2}\left(r_{0}^{2}+4 \epsilon\right)}{8 r_{0}^{6} \epsilon^{3}} \\
& +\frac{\xi^{2}\left(3 r_{0}^{6}-26 r_{0}^{2} \epsilon^{2}+4 \epsilon^{3}\right)}{16 r_{0}^{6} \epsilon^{3}} \\
& +\frac{\xi\left(-15 r_{0}^{6}+4 r_{0}^{4} \epsilon+102 r_{0}^{2} \epsilon^{2}+8 \epsilon^{3}\right)}{240 r_{0}^{6} \epsilon^{3}} \\
& +\frac{22 r_{0}^{6}-20 r_{0}^{4} \epsilon-107 r_{0}^{2} \epsilon^{2}+58 \epsilon^{3}}{3360 r_{0}^{6} \epsilon^{3}} \\
& s_{r}^{r}=\frac{17 r_{0}^{6}-74 r_{0}^{4} \epsilon-20 r_{0}^{2} \epsilon^{2}+524 \epsilon^{3}}{6720 r_{0}^{6} \epsilon^{3}}
\end{aligned}
$$




$$
\begin{gathered}
v_{r}^{r}=-\frac{-66 r_{0}^{6}+228 r_{0}^{4} \epsilon+223 r_{0}^{2} \epsilon^{2}-1490 \epsilon^{3}}{3360 r_{0}^{6} \epsilon^{3}}, \\
t_{\theta}^{\theta}=-\frac{\xi^{3}\left(23 r_{0}^{6}-96 r_{0}^{4} \epsilon+36 r_{0}^{2} \epsilon^{2}+128 \epsilon^{3}\right)}{16 r_{0}^{6} \epsilon^{3}} \\
+\frac{\xi^{2}\left(87 r_{0}^{6}-280 r_{0}^{4} \epsilon-44 r_{0}^{2} \epsilon^{2}-136 \epsilon^{3}\right)}{32 r_{0}^{6} \epsilon^{3}} \\
+\frac{\xi\left(-441 r_{0}^{6}+1328 r_{0}^{4} \epsilon+460 r_{0}^{2} \epsilon^{2}+1016 \epsilon^{3}\right)}{480 r_{0}^{6} \epsilon^{3}} \\
-\frac{-283 r_{0}^{6}+833 r_{0}^{4} \epsilon+382 r_{0}^{2} \epsilon^{2}+538 \epsilon^{3}}{3360 r_{0}^{6} \epsilon^{3}}, \\
s_{\theta}^{\theta}=\frac{-143 r_{0}^{6}+224 r_{0}^{4} \epsilon+382 r_{0}^{2} \epsilon^{2}+1560 \epsilon^{3}}{13440 r_{0}^{6} \epsilon^{3}}
\end{gathered}
$$

and

$$
v_{\theta}^{\theta}=\frac{-95 r_{0}^{6}+175 r_{0}^{4} \epsilon+416 r_{0}^{2} \epsilon^{2}+106 \epsilon^{3}}{1120 r_{0}^{6} \epsilon^{3}} .
$$

The simplicity of the metric is reflected in the simplicity of the resulting stress-energy tensors. Analysis of the energy density and the radial tension of the spinor and vector fields indicates that the Morris-Thorne conditions cannot be satisfied. Specifically, the energy density is negative for $\varepsilon<0.3084$ and $\varepsilon<0.3153$, for massive spinors and vectors, respectively. The radial pressure is always negative. On the other hand, the quantized massive scalar field has three regions in the parameter space in which the Morris-Thorne conditions are satisfied. It should be noted however that neither minimal nor conformal couplings belong to these regions.

\section{F. Wormhole supported by phantom energy}

Finally, we shall analyze the wormhole model proposed by Zaslavskii [28]. In this model the phantom energy supports the wormhole described by the line element (1) with

$$
\phi(r)=\frac{1}{2} \ln \frac{r_{1}}{r} \quad \text { and } \quad b(r)=r_{0}+d\left(r-r_{0}\right)
$$

for $r_{0} \leq r \leq r_{b}$ and the Schwarzschild metric for $r>r_{b}$. Here $r_{1}$ is some constant that affects the normalization of time (our final results do not depend on it) and $d<1$. Let us observe that the wormhole is not asymptotically flat. It can, however, be matched to the external Schwarzschild 
spacetime at some fixed radius. The stress-energy tensor of the scalar, spinor and vector fields is given by Eq. (10) with

$$
\begin{aligned}
& t_{t}^{t}=\frac{(9 d-11)(1+3 d)^{2} \xi^{3}}{8 r_{0}^{6}}-\frac{(1+3 d)\left(57 d^{2}-84 d+19\right) \xi^{2}}{16 r_{0}^{6}} \\
& +\frac{\left(735 d^{3}-1113 d^{2}+277 d+5\right) \xi}{240 r_{0}^{6}}-\frac{823 d^{3}-1391 d^{2}+537 d-97}{3360 r_{0}^{6}} \\
& s_{t}^{t}=\frac{786 d^{3}-2519 d^{2}+2792 d-899}{6720 r_{0}^{6}}, \\
& v_{t}^{t}=\frac{4013 d^{3}-11325 d^{2}+11283 d-3587}{3360 r_{0}^{6}}, \\
& t_{r}^{r}=\frac{(3 d-5)(1+3 d)^{2} \xi^{3}}{8 r_{0}^{6}}-\frac{(1+3 d)\left(15 d^{2}-24 d+1\right) \xi^{2}}{16 r_{0}^{6}} \\
& +\frac{\left(63 d^{3}-97 d^{2}+5 d-3\right) \xi}{80 r_{0}^{6}}-\frac{263 d^{3}-495 d^{2}+201 d-97}{3360 r_{0}^{6}}, \\
& s_{r}^{r}=\frac{-208 d^{3}+631 d^{2}-638 d+375}{6720 r_{0}^{6}}, \\
& v_{r}^{r}=\frac{-915 d^{3}+2227 d^{2}-1709 d+781}{3360 r_{0}^{6}}, \\
& t_{\theta}^{\theta}=\frac{(27 d-19)(1+3 d)^{2} \xi^{3}}{16 r_{0}^{6}}-\frac{(1+3 d)\left(153 d^{2}-158 d+37\right) \xi^{2}}{32 r_{0}^{6}} \\
& +\frac{\left(2037 d^{3}-2391 d^{2}+655 d+83\right) \xi}{480 r_{0}^{6}}-\frac{1411 d^{3}-2273 d^{2}+1265 d-147}{3360 r_{0}^{6}}, \\
& s_{\theta}^{\theta}=-\frac{1437 d^{3}-4419 d^{2}+4811 d-1225}{13440 r_{0}^{6}}
\end{aligned}
$$

and

$$
v_{\theta}^{\theta}=-\frac{2791 d^{3}-5573 d^{2}+3949 d-399}{3360 r_{0}^{6}} .
$$




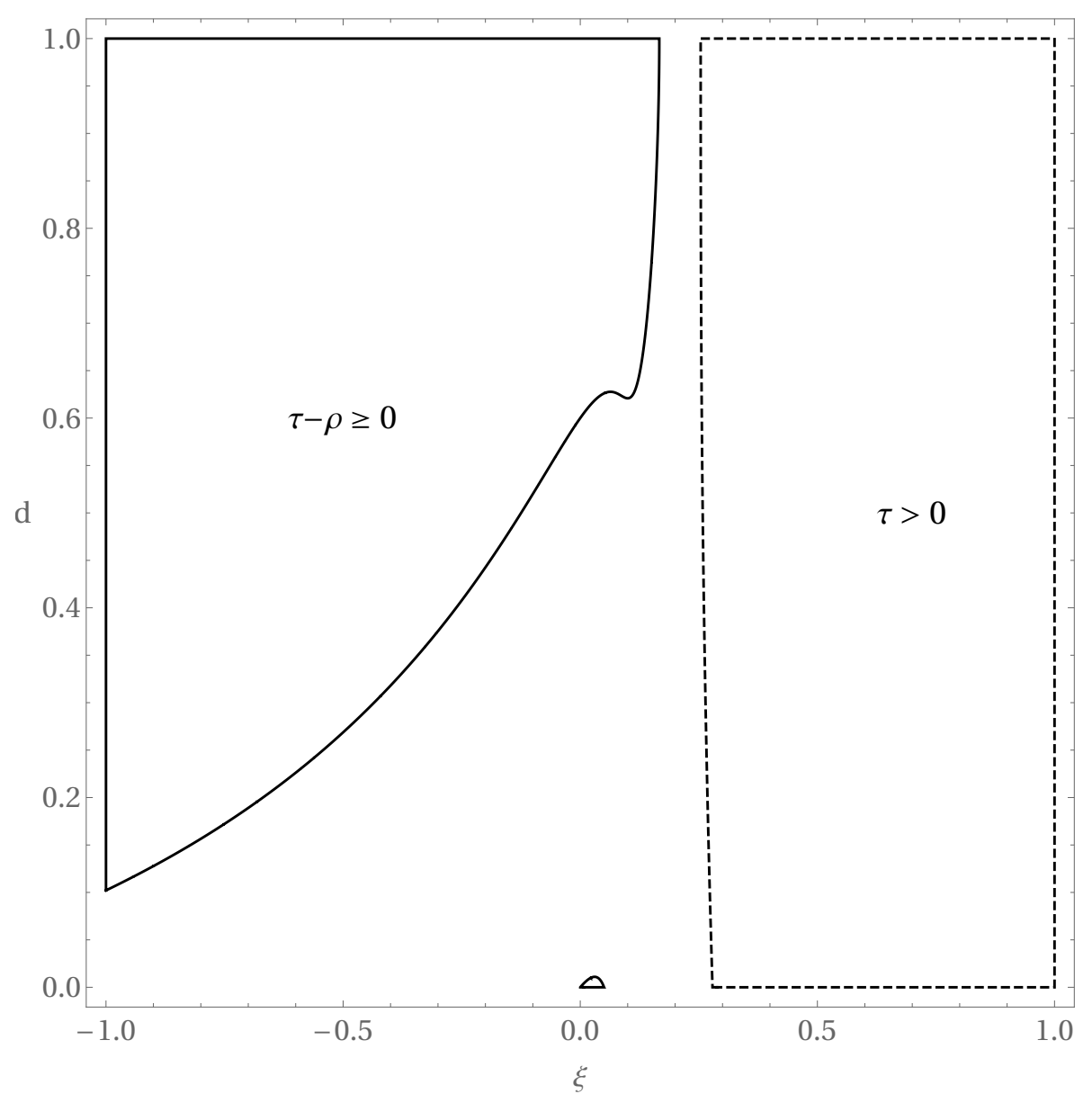

FIG. 1: The regions of the rectangle $-1<\xi<1,0<d<1$ for which the exoticity condition is satisfied (solid line). The radial tension is positive within the region bouded by a dashed line.

Let us consider the stress-energy tensor of the quantized spinor and vector fields first. The energy density is positive for $d<0.5428$ for massive spinors and for $d<0.6139$ for vectors. The radial tension is always negative and the second Morris-Thorne condition is violated. Thus there are no configurations that sustain a Zaslavskii wormhole. The parameter space for the massive scalar field is two-dimensional. Simple analysis shows that there are no configurations that satisfy the Morris-Thorne conditions as can easily be inferred form Fig. 1. (The regions of the parameter space defined by the Morris-Thorne conditions are disjoint.)

\section{FINAL REMARKS}

In this paper, we have attempted to answer the question posed in Ref. [7] of whether the stressenergy tensor of the quantized massive fields has the appropriate form to support the traversable wormhole. The scalar case has been considered earlier by Taylor, Hiscock and Anderson (cases A 
- E). Our results are in concord with the previous studies and indicate that the quantized massive scalar, spinor and vector fields do not satisfy simultaneously the Morris-Thorne conditions. Here we adopted a somewhat conservative point of view and treated as physical only the minimal and conformal curvature couplings. The reported calculations indicate serious problems one encounters in the maintenance of a traversable wormhole. Indeed, regardless of the spin, the quantum fields tend to destroy a wormhole and this may suggest that even if the fields with the desired properties exist, the net effect would be negative. It is simply because the fields that do not satisfy the Morris-Thorne conditions would also be present there. We have borrowed the last argument from Ref. [7]. This, of course, does not exclude the possibility of the existence of the wormholes as self-consistent solutions of the semiclassical Einstein field equations

$$
G_{a b}+\alpha I_{a b}+\beta J_{a b}=8 \pi\left\langle T_{a b}\right\rangle
$$

with the quadratic terms $I_{a b}$ and $J_{a b}$, the coupling constants (usually set to zero) $\alpha, \beta$ and with the source term given by the stress-energy tensor of the quantized fields functionally depending on the metric. In this case, however, we are looking for the particular solution (or a class of solutions that satisfy prescribed requirements) of the system of the differential equations. For the stress-energy tensor constructed within the framework of the Schwinger-DeWitt approximation, the problem reduces to a complicated system of differential equations, which must be studied numerically. To be more precise, let us consider the line element describing a wormhole in the form

$$
d s^{2}=-f(l) d t^{2}+d l^{2}+r^{2}(l)\left(d \theta^{2}+\sin ^{2} \theta d \phi^{2}\right),
$$

where $l$ is the proper distance from the throat and $f$ and $r$ are two unknown functions. The resulting $(l l)$-component of the semiclassical Einstein field equations is of the fifth order, whereas the remaining ones are of the sixth order. These equations, with carefully chosen initial conditions, can be analyzed numerically in the spirit of Ref. [17].

The results presented in this paper raise the interesting question of whether obstacles one encounters in constructing and maintaining the traversable wormhole are specific to the fourdimensional world or generic. The techniques described here may shed light on this problem. Indeed, since the Schwinger-DeWitt approach can be extended to other dimensions [25]27] (see also Refs. [31, 33] ) one can obtain valuable information regarding the general nature of the traversable wormholes. Indeed, let us concentrate on the quantized massive scalar field. For $D=4$ and $D=5$ the effective action $W^{(1)}$ is constructed from the coefficient $\left[a_{3}\right]$ whereas for $D=6$ and $D=7$ the coefficient $\left[a_{4}\right]$ is needed. The stress-energy tensor can be obtained in a standard way, i.e., by 
taking the functional derivative of the action with respect to the metric and the resulting general formulas can be used in the spacetime of the higher-dimensional wormhole. As the computational complexity of the problem rapidly grows with the dimension $D$, the (extremely complicated and time-consuming) calculations are practically limited to $D \leq 9$. All these problems are under active investigation and we intend to return to them in the future.

\section{Acknowledgments}

Discussions with Paweł Sadurski are gratefully acknowledged. J.M. was partially supported by the Polish National Science Centre grant no. DEC-2014/15/B/ST2/00089.

[1] M. S. Morris and K. S. Thorne, Am. J. Phys. 56, 395 (1988).

[2] M. S. Morris, K. S. Thorne, and U. Yurtsever, Phys. Rev. Lett. 61, 1446 (1988).

[3] M. Visser, Lorentzian wormholes: From Einstein to Hawking (American Institute of Physics, Woodbury, NY, 1995).

[4] F. S. N. Lobo, Int. J. Mod. Phys. D25, 1630017 (2016).

[5] K. A. Bronnikov and S. G. Rubin, Black Holes, Cosmology and Extra Dimensions (World Scientific, Singapore, 2012).

[6] E. Ayon-Beato, F. Canfora, and J. Zanelli, Physics Letters B 752, 201 (2016).

[7] B. E. Taylor, W. A. Hiscock, and P. R. Anderson, Phys. Rev. D55, 6116 (1997).

[8] P. R. Anderson, W. A. Hiscock, and D. A. Samuel, Phys. Rev. D51, 4337 (1995).

[9] E. D. Carlson, P. R. Anderson, A. Fabbri, S. Fagnocchi, W. H. Hirsch, and S. Klyap, Phys. Rev. D82, 124070 (2010).

[10] V. Khatsymovsky, Phys. Lett. B399, 215 (1997).

[11] A. A. Popov, Grav. Cosmol. 20, 203 (2014).

[12] V. B. Bezerra, E. R. Bezerra de Mello, N. R. Khusnutdinov, and S. V. Sushkov, Phys. Rev. D81, $084034(2010$.

[13] A. A. Popov and S. V. Sushkov, Phys. Rev. D63, 044017 (2001).

[14] A. A. Popov, Phys. Rev. D64, 104005 (2001).

[15] N. R. Khusnutdinov, Phys. Rev. D67, 124020 (2003).

[16] R. Garattini and F. S. N. Lobo, Class. Quant. Grav. 24, 2401 (2007).

[17] D. Hochberg, A. Popov, and S. V. Sushkov, Phys. Rev. Lett. 78, 2050 (1997).

[18] B. S. DeWitt, Dynamical Theory of groups and fields (Gordon and Breach, New York, 1965).

[19] I. G. Avramidi, Heat kernel and quantum gravity (Springer-Verlag, Berlin, 2000).

[20] V. P. Frolov and A. Zelnikov, Phys.Rev. D29, 1057 (1984). 
[21] J. Matyjasek, Phys. Rev. D61, 124019 (2000).

[22] J. Matyjasek, Phys. Rev. D63, 084004 (2001).

[23] B. E. Taylor, W. A. Hiscock, and P. R. Anderson, Phys. Rev. D61, 084021 (2000).

[24] J. Matyjasek and D. Tryniecki, Phys. Rev. D79, 084017 (2009).

[25] J. Matyjasek, Phys. Rev. D94, 084048 (2016).

[26] J. Matyjasek and P. Sadurski, Phys. Rev. D92, 044023 (2015).

[27] J. Matyjasek and P. Sadurski, Phys. Rev. D91, 044027 (2015).

[28] O. B. Zaslavskii, Phys. Rev. D72, 061303 (2005.

[29] J. A. M. Vermaseren (2000), arXiv:math-ph/0010025.

[30] B. Ruijl, T. Ueda, and J. Vermaseren (2017), arXiv:1707.06453.

[31] R. T. Thompson and J. P. S. Lemos, Phys. Rev. D80, 064017 (2009).

[32] P. Taylor and C. Breen, Phys. Rev. D94, 125024 (2016).

[33] P. Taylor and C. Breen (2017), arXiv:1709.00316. 